\title{
A DLL based clock multiplier using rotational DCDL and PRNG for spur reduction
}

\author{
Jinseop Noh and Dong-Woo Jee ${ }^{\text {a) }}$ \\ Department of Electrical and Computer Engineering, Ajou University, \\ Suwon, Korea \\ a)dwjee@ajou.ac.kr
}

Abstract: This paper presents a DLL based clock multiplier with a novel spur reduction technique. By randomly selecting delay line with pseudo random number generator (PRNG), the proposed scheme reduces the output spur due to delay cell mismatches. Rotational digitally controlled delay line (DCDL) is also proposed for seamless generation of clock edges even at random delay line switching. The clock multiplier is designed in $0.18 \mu \mathrm{m}$ CMOS process and achieves $5 \sim 11 \mathrm{~dB}$ reduction of spur while consuming $169.4 \mu \mathrm{W}$ for $16 \mathrm{MHz}$. The core area is $0.608 \mathrm{~mm}^{2}$.

Keywords: DLL, digital DLL, clock multiplier, spur reduction, PRNG

Classification: Integrated circuits

\section{References}

[1] G. Chien and P. R. Gray: "A 900-MHz local oscillator using DLL-based frequency multiplier technique for PCS applications," IEEE J. Solid-State Circuits 35 (2000) 1996 (DOI: 10.1109/4.890315).

[2] Y. Ogasawara, et al.: "An $18 \mu \mathrm{W}$ spur canceled clock generator for recovering receiver sensitivity in wireless SoCs," 2016 IEEE Symp. VLSI Circuits (2016) (DOI: 10.1109/VLSIC.2016.7573484).

[3] S. L. J. Gierkink: "Low-spur, low-phase-noise clock multiplier based on a combination of PLL and recirculating DLL with dual-pulse ring oscillator and self-correcting charge pump," IEEE J. Solid-State Circuits 43 (2008) 2967 (DOI: 10.1109/JSSC.2008.2006225).

[4] J. G. Maneatis: "Low-jitter process-independent DLL and PLL based on selfbiased techniques," IEEE J. Solid-State Circuits 31 (1996) 1723 (DOI: 10. 1109/JSSC.1996.542317).

[5] B. He, et al:: "A wide-range edge-combining DLL with a charge pump for low spur," 2011 IEEE Ninth International Conference on Dependable, Autonomic and Secure Computing (DASC) (2011) 52 (DOI: 10.1109/DASC.2011.33).

[6] K. Ryu, et al.: "High-speed, low-power, and highly reliable frequency multiplier for DLL-based clock generator," IEEE Trans. Very Large Scale Integr. (VLSI) Syst. 24 (2016) 1484 (DOI: 10.1109/TVLSI.2015.2453366).

[7] O. Casha, et al: "Analysis of the spur characteristics of edge-combining DLLbased frequency multipliers," IEEE Trans. Circuits Syst. II, Exp. Briefs 56 (2009) 132 (DOI: 10.1109/TCSII.2008.2011605).

[8] H. Guo, et al:: "Spur analysis and reduction of edge combining DLL-based frequency multiplier," 2013 26th IEEE Canadian Conference of Electrical and Computer Engineering (2013) (DOI: 10.1109/CCECE.2013.6567802). 
[9] F. R. Liao and S. S. Lu: "A programmable edge-combining DLL with a current-splitting charge pump for spur suppression," IEEE Trans. Circuits Syst. II, Exp. Briefs 57 (2010) 946 (DOI: 10.1109/TCSII.2010.2087992).

[10] S.-K. Lee, et al.: "A $1.3 \mu \mathrm{W} 0.6 \mathrm{~V}$ 8.7-ENOB successive approximation ADC in a $0.18 \mu \mathrm{m}$ CMOS," IEEE Symp. VLSI Circuits (2009) 242.

[11] M. Hassani and S. Saeedi: "Edge-combining multi-phase DLL frequency multiplier with reduced static phase offset and linearized delay transfer curve," Analog Integr. Circuits Signal Process. 82 (2015) 705 (DOI: 10.1007/s10470015-0495-1).

[12] J. Lim, et al.: "Toward a reduced-wire readout system for ultrasound imaging." 2014 36th Annual Int. Conf. IEEE Engineering in Medicine and Biology Society (2014) 5080 (DOI: 10.1109/EMBC.2014.6944767).

[13] D.-W. Jee: "Robust high-multiplication factor MDLL using IIR filter-based accumulated jitter reduction," Electron. Lett. 54 (2018) 743 (DOI: 10.1049/el. 2018.1091).

\section{Introduction}

The clock multiplier is an important building block in clock/frequency generation in wireless communication systems and System-on-chip (SoC) [1, 2]. A phase locked loop (PLL) is widely used architecture for clock multiplication. However, PLL is basically two pole system, one from the loop filter and the other from the oscillator, and so there is a tradeoff between bandwidth and stability. Due to the limited choice of loop bandwidth, the PLL has an inevitable issue of jitter accumulation [3]. On the other hand, the delay locked loop (DLL) is a single pole system since the loop filter voltage instantly change the output delay. Compare to PLL, therefore, the DLL typically has less jitter accumulation and less settling time. $[4,5]$.

The DLL-based clock multiplication uses an edge-combining technique [6] from multiple clock edges at the delay chain. Therefore, any mismatches in delay cell due to process variation and inadequate layout design cause spurious tone at output $[7,8]$. Assuming the delay of one delay cell is longer than that of the others, this delay difference appears in the output waveform with a period of the reference clock. In frequency domain, the spur will appear at the offset frequency corresponding to the harmonics of the reference frequency around the output frequency. Static phase offset due to current mismatch of charge pump in analog DLL also contributes to the spur level.

Several researches have reported spur reduction techniques for DLL-based clock multiplier [7, 8, 9]. In [9], a current-splitting charge-pump is adopted to reduce the reference spur by the static phase offset, resulting reference spur level below $-39.2,-36.8$, and $-26.2 \mathrm{dBc}$ for $\times 2, \times 3, \times 6$ clock multiplication, respectively. In this paper, we introduce delay line scrambling technique to reduce the spur levels due to delay cell mismatches. This paper is organized as follows. In section 2, we describe the proposed DLL-based clock multiplier and circuit implementations. The section 3 and 4 present implementation results and conclusion, respectively. 


\section{Proposed architecture}

The block diagram of the proposed clock multiplier is shown in Fig. 1. Reference clock $\left(f_{\text {ref }}\right)$ is injected to rotational digitally controlled delay line (DCDL) for multiple clock edge generation. Pseudo random number generator (PRNG) based DCDL decoder randomly selects delay line in rotational DCDL to shape delay cell mismatches. Matrix switch performs reordering of output phases for edge combiner which finally generates multiplier clock, $f_{\text {out }}$. For $\times 16$ clock multiplication, 16 output phases $\left(\Phi_{01 \sim 16}\right)$ are applied to edge combiner. Phase output $\Phi_{017}$ exhibits the same phase of $\Phi_{\mathrm{o} 1}$, and is used for phase detector (PD). PD [10] generates $1 \mathrm{~b}$ up/ down signal for digital loop filter (DLF) operation. This digital DLL implementation eliminates the charge pump and minimizes the spur from the static phase offset.

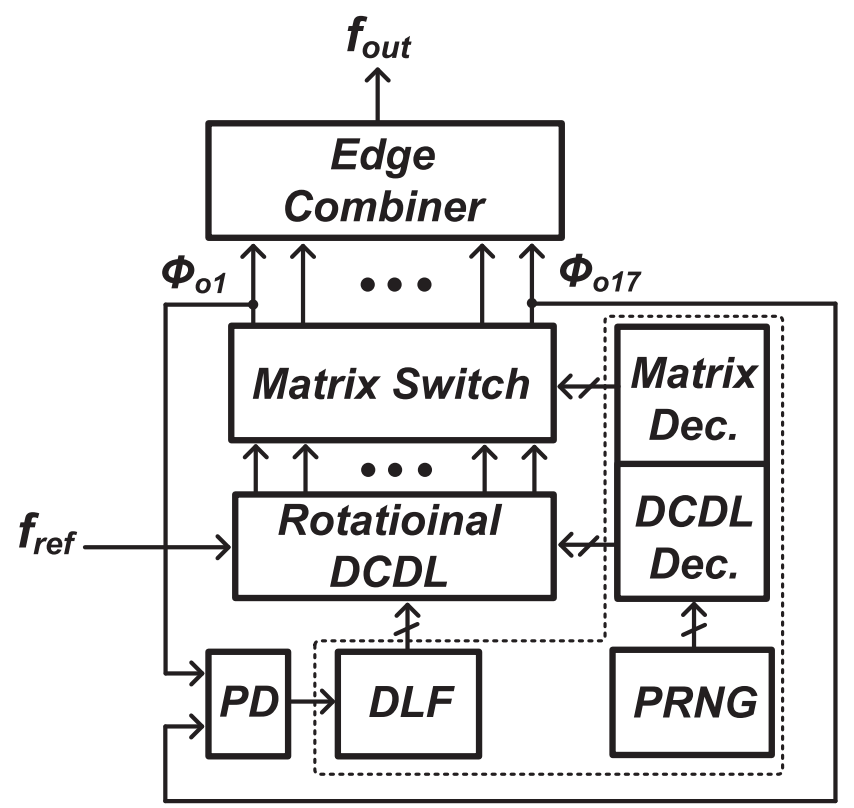

Fig. 1. Top block diagram of proposed DLL-based clock multiplier

\subsection{Rotational DCDL and matrix switch}

The proposed rotational DCDL consists of a ring-shaped rotational delay line composed of 18 delay cells, 18 branch delay lines composed of 4 delay cells, and control switches, as shown in Fig. 2(a). Each delay cell is controlled by the control voltage from $12 \mathrm{~b}$ digital to analog converter (DAC), described in the section 2.3. Switch control signal $\left(S_{e} l_{D C D L}\right)$ from DCDL decoder decides $f_{r e f}$ injection points and one of the 18 branch delay line. For example, if $f_{\text {ref }}$ is applied to the input of the $3^{\text {rd }}$ delay cell $\left(d_{3}\right)$, the $15^{\text {th }}$ branch delay line $\left(d_{15_{-} \sim 4}\right)$ is selected for total 17 output clock edge generation (Fig. 2(b)). If DCDL decoder selects the $10^{\text {th }}$ delay cell $\left(d_{10}\right)$ for $f_{\text {ref }}$ injection point, the $4^{\text {th }}$ branch delay line $\left(d_{4_{-} 1 \sim 4}\right)$ is used for output phase generation. Therefore, total 18 delay line configurations are possible with designed rotational DCDL. Note that unselected branch lines are turned off to save power consumption.

Random selection of one of 18 delay lines scrambles mismatches in delay cells, 
larger delay than other delay cells and $f_{\text {ref }}$ is always applied to the input of the $3^{\text {rd }}$ delay cell $\left(d_{3}\right)$ as in case of Fig. 2(b), $10^{\text {th }}$ clock edge of output clock always shows more delay than others. Since such delay change is repeated in every $f_{\text {ref }}$ period ( $\mathrm{T}_{\text {ref }}$ ), it is the source of spurs at the harmonic offsets of $f_{\text {ref }}$ in output spectrum. However, if $f_{\text {ref }}$ is injected to $d_{10}$ in next cycle, in case of Fig. $2(\mathrm{c}), 3^{\text {rd }}$ clock edge of output clock shows more delay than others and the periodicity of delay change is no longer $\mathrm{T}_{\text {ref }}$. In other words, although delay cell mismatches still exist in the delay line, the periodicity of delay change is scrambled with the proposed randomized delay line selection and the spur power at the harmonic offsets of $f_{\text {ref }}$ are also spread out.

All the outputs of delay cells in DCDL are connected to matrix switch since the points at which the clock edges are output are different for each delay line selection. With a control from matrix decoder, matrix switch rearranges and outputs 17 phases for each selection. For example, in case of Fig. 2(b), output of $d_{3}$ and $d_{15 \_}$are used for $\Phi_{o 1}$ and $\Phi_{o 17}$, respectively, while output of $d_{10}$ and $d_{4 \_}$are used for $\Phi_{o 1}$ and $\Phi_{017}$, respectively, in case of Fig. 2(c).

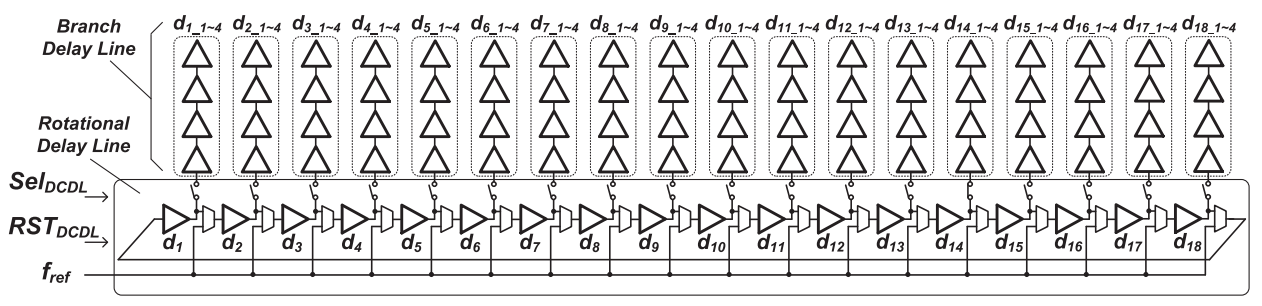

(a)

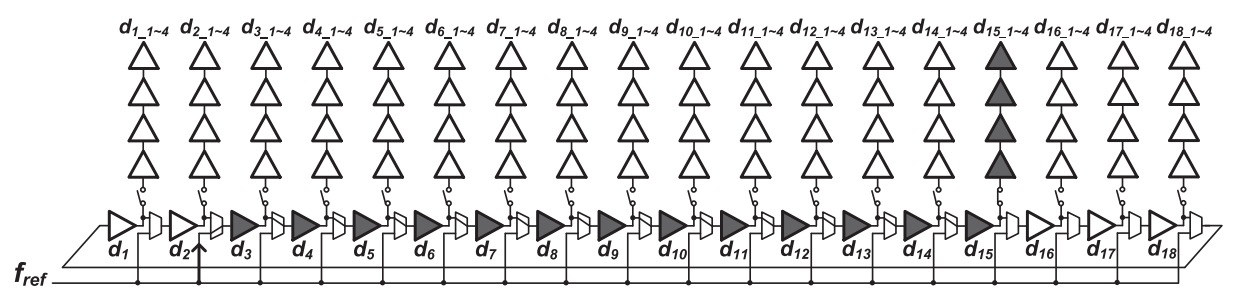

(b)

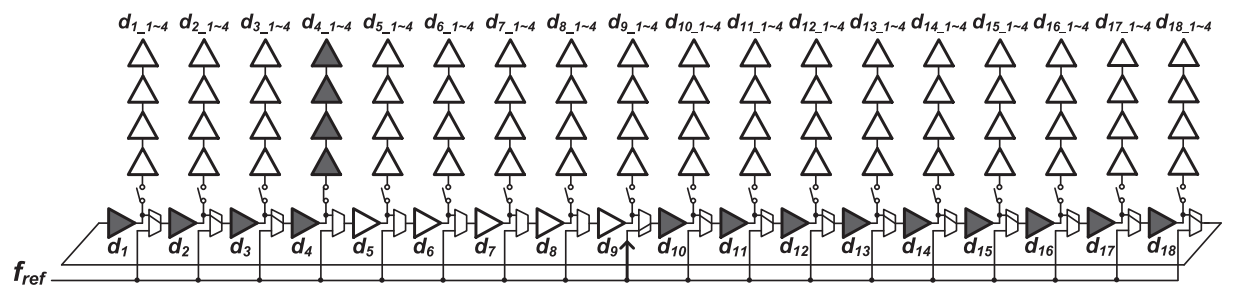

(c)

Fig. 2. (a) Proposed rotational DCDL architecture (b) when $f_{\text {ref }}$ is applied to $d_{3}$ (c) when $f_{\text {ref }}$ is applied to $d_{10}$

Fig. 3 shows the timing diagram of rotational DCDL where $f_{\text {ref }}$ injection point is changed from $d_{3}$ to $d_{10}$. After $f_{\text {ref }}$ is applied to $d_{3}, 13$ clock edges are output from delay cell outputs $\left(d_{30 \sim 15 o}\right)$ in order. The next 4 clock edges are then output ( $\left.d_{15_{-} 10 \sim 15_{-} 4 o}\right)$ from the branch delay line connected to the $d_{15}$ output. The rotational delay cells $\left(d_{1 \sim 18}\right)$ are reset $\left(R S T_{D C D L}\right)$ in synchronization with the first branch

๑) IEICE 2019

DOI: $10.1587 /$ elex.16.20181022 Received November 14, 2018 Accepted December 3, 2018 Publicized January 22, 2019 Copyedited February 10, 2019 
delay cell output (in this case, $d_{15_{-} 10}$ ), and $S e l_{D C D L}$ also moves to next injection point (in this case, $d_{10}$ ). Since rotational delay line is reset to low value before $f_{\text {ref }}$ arrives, switching to any delay line configuration is possible regardless of $f_{\text {ref }}$ duty cycle. At the same time, since the remaining clock phases are output in the selected branch line, which are not reset, total 17 phases for clock multiplication and phase detection are continuously generated.

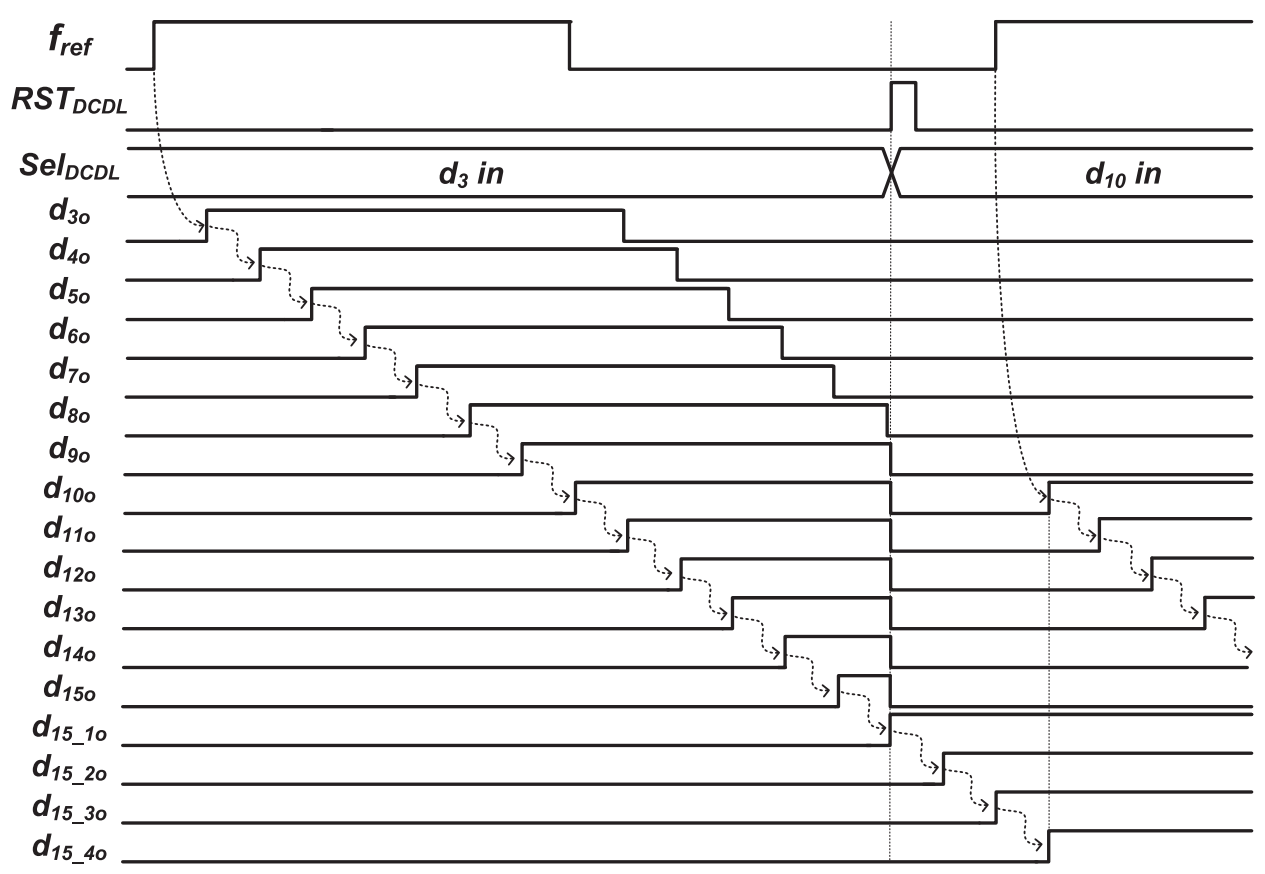

Fig. 3. Timing diagram of rotational DCDL where $f_{\text {ref }}$ injection point is changed from $d_{3}$ to $d_{10}$

\subsection{PRNG}

The PRNG consists of $8 \mathrm{~b}$ linear feedback shift register (LFSR) for sufficiently long pseudo random codes, and delta sigma modulator (DSM) for output bit conditioning and further randomization (Fig. 4). The PRNG output (OUT ${ }_{\mathrm{PRNG}}$ ) has a digital output of $5 \mathrm{~b}$ for total 18 delay lines selection. To provide only 18 selection code out of 32 possible binary $5 \mathrm{~b}$ representation, 18 level quantization (right side table in Fig. 4) is adopted at DSM quantizer.

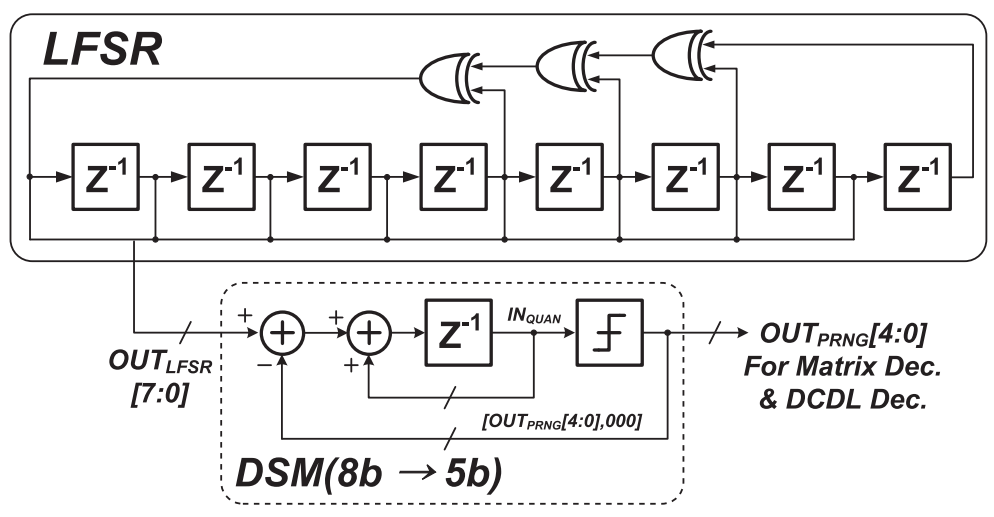

\begin{tabular}{|c|c|}
\hline IN $_{\text {QUAN }}$ & $\begin{array}{c}\text { OU } \\
{[4: 0]}\end{array}$ \\
\hline $0 \sim 14$ & 00000 \\
\hline $0 \sim 14$ & 00001 \\
\hline $15 \sim 29$ & 00010 \\
\hline $30 \sim 44$ & 00011 \\
\hline $45 \sim 59$ & 00011 \\
\hline $60 \sim 74$ & 00100 \\
\hline $75 \sim 89$ & 00101 \\
\hline $90 \sim 104$ & 00110 \\
\hline $105 \sim 119$ & 00111 \\
\hline $120 \sim 134$ & 01000 \\
\hline $135 \sim 149$ & 01001 \\
\hline $150 \sim 164$ & 01010 \\
\hline $165 \sim 179$ & 01011 \\
\hline $180 \sim 194$ & 01100 \\
\hline $195 \sim 209$ & 01101 \\
\hline $210 \sim 224$ & 01110 \\
\hline $225 \sim 239$ & 01111 \\
\hline $240 \sim 254$ & 10000 \\
\hline $255 \sim 270$ & 10001 \\
\hline
\end{tabular}

Fig. 4. Circuit diagram of PRNG 


\subsection{DLF and DAC}

Fig. 5(a) shows the DLF that consists of $12 \mathrm{~b}$ integrator, a binary-to-thermometer code converter (B2T) and DSM. After integrating 1b PD output with $1 \mathrm{MHz}$ $\left(=f_{\text {ref }}\right)$ clock, the 4 most significant bits (MSBs) of $12 \mathrm{~b}$ integrator output are directly applied to DAC for coarse tuning. Next 4 bits are converted to thermometer code to improve overall linearity of DAC, and used for fine tuning. The 4 least significant bits (LSBs) are converted to $1 \mathrm{~b}$ stream with DSM with $16 \mathrm{MHz}$ clock $\left(=f_{\text {out }}\right)$ to further improve DAC resolution. Circuit diagram of DAC is shown in Fig. 5(b).

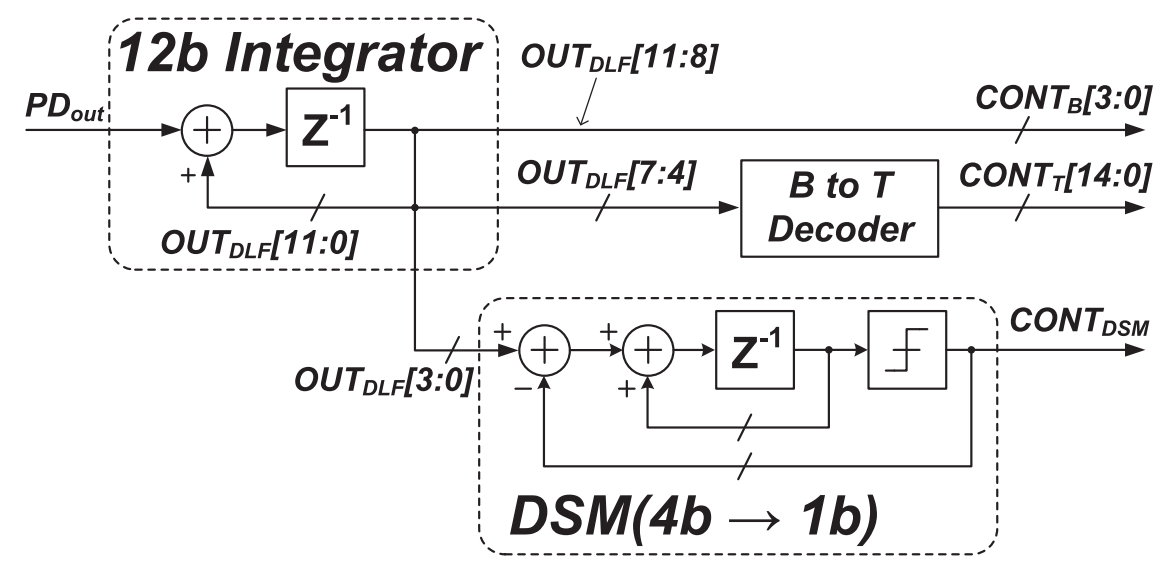

(a)

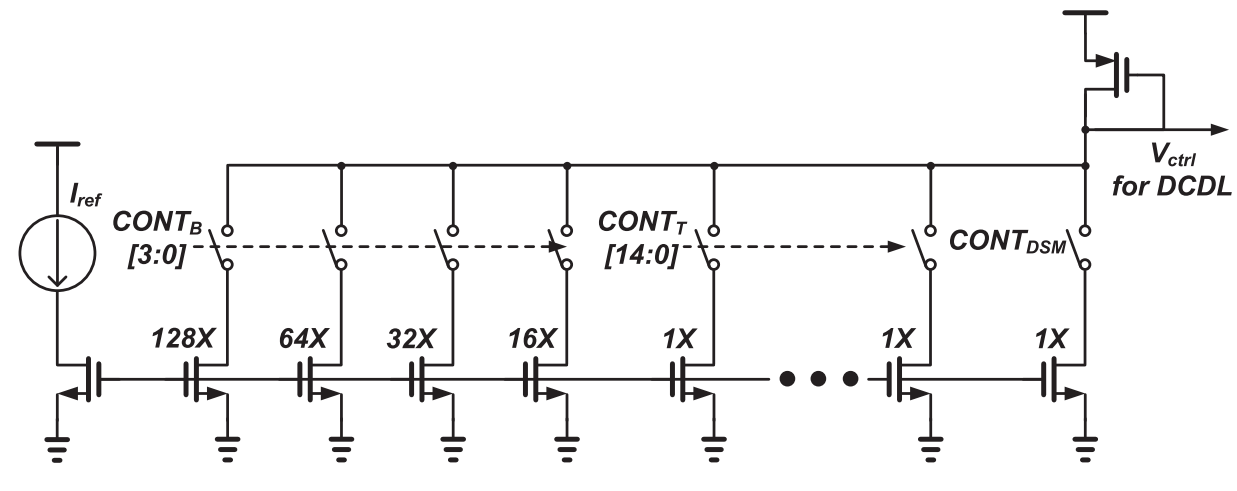

(b)

Fig. 5. The circuit diagram of (a) DLF, and (b) DAC in rotational DCDL

\section{Post layout results}

The proposed clock multiplier was designed in a $0.18 \mu \mathrm{m}$ standard CMOS process and post layout verification was performed after RC parasitic extraction. Output frequency is $16 \mathrm{MHz}$ with $1 \mathrm{MHz}$ reference clock for low power sensor SoC application. Fig. 6 shows a chip layout which has a core area of $950 \mu \mathrm{m} \times 640 \mu \mathrm{m}$ $\left(=0.6 \mathrm{~mm}^{2}\right)$. The total power consumption is $169.4 \mu \mathrm{W}$.

Fig. 7 shows the output spectrum of the proposed clock multiplier. For performance comparison, intentional $1.5 \%$ mismatches are applied to delay cells, which generates $-31.8 \mathrm{dBc}$ worst case spur. In order to verify the validity of the proposed scheme more accurately, DCDL noise component obtained from phase 
noise simulation result $(-83.5 \mathrm{dBc} / \mathrm{Hz} @ 1 \mathrm{MHz}$, Fig. 8) is also included. The result shows that spur levels due to the delay cell mismatch are improved by $5 \sim 11 \mathrm{~dB}$ and now close to the spur levels without mismatch that is limited by the coupling effect from device parasitic capacitance in multiplexer switches for $f_{\text {ref }}$ injection. The worst case spur level is improved from $31.8 \mathrm{dBc}$ to $38 \mathrm{dBc}$. The RMS and peak-to-peak jitter are $1.1 \mathrm{~ns}$ and $21.5 \mathrm{~ns}$, respectively.

In order to apply the proposed technique to the conventional edge-combining DLL-based clock multiplier, PRNG, decoders, the matrix switch and branch delay lines are additionally required. However, additional power consumption is only $14.78 \mu \mathrm{W}(8.94 \mu \mathrm{W}$ from the matrix switch and $5.84 \mu \mathrm{W}$ from PRNG \& decoders). Since only 17 delay cells are turned on for each delay line selection, branch delay lines does not consume any additional power. The layout areas of the matrix switch, PRNG \& decoders, and branch delay lines are $0.05 \mathrm{~mm}^{2}, 0.03 \mathrm{~mm}^{2}, 0.182 \mathrm{~mm}^{2}$, respectively. Table I summaries the performance comparisons with the state-of-the art edge combining DLLs.

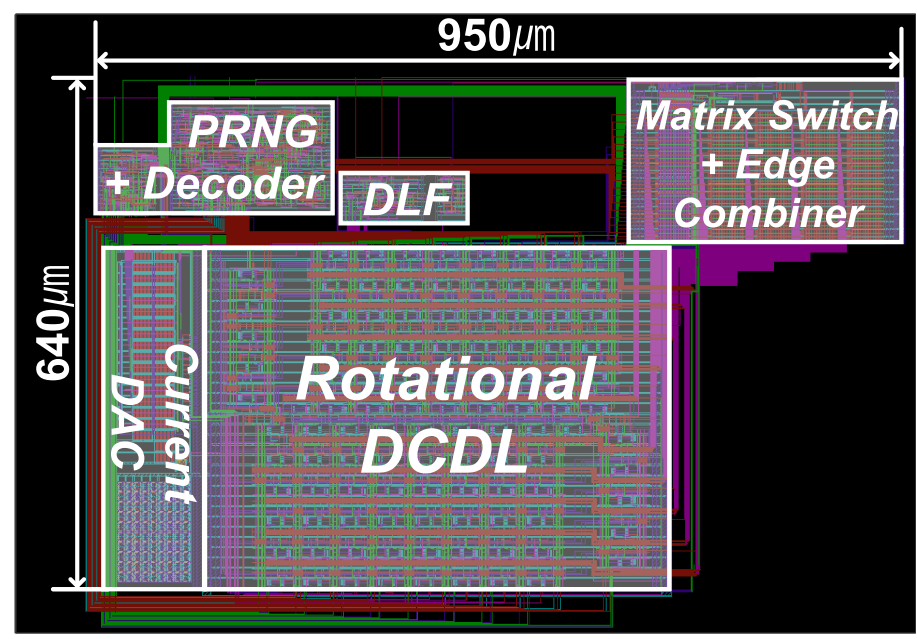

Fig. 6. Chip layout

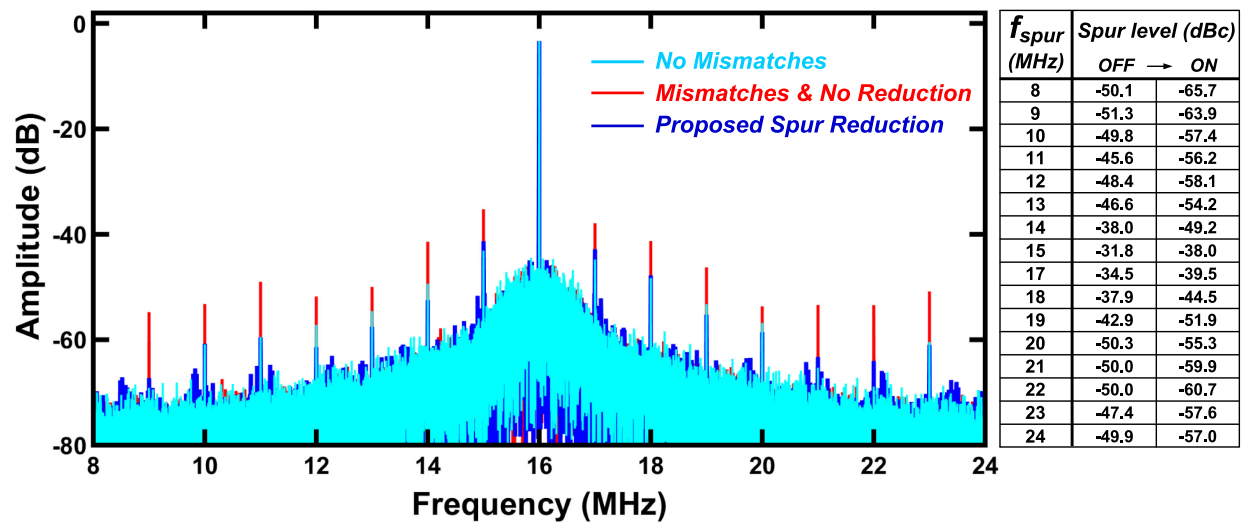

Fig. 7. The output spectrum of the proposed clock multiplier 


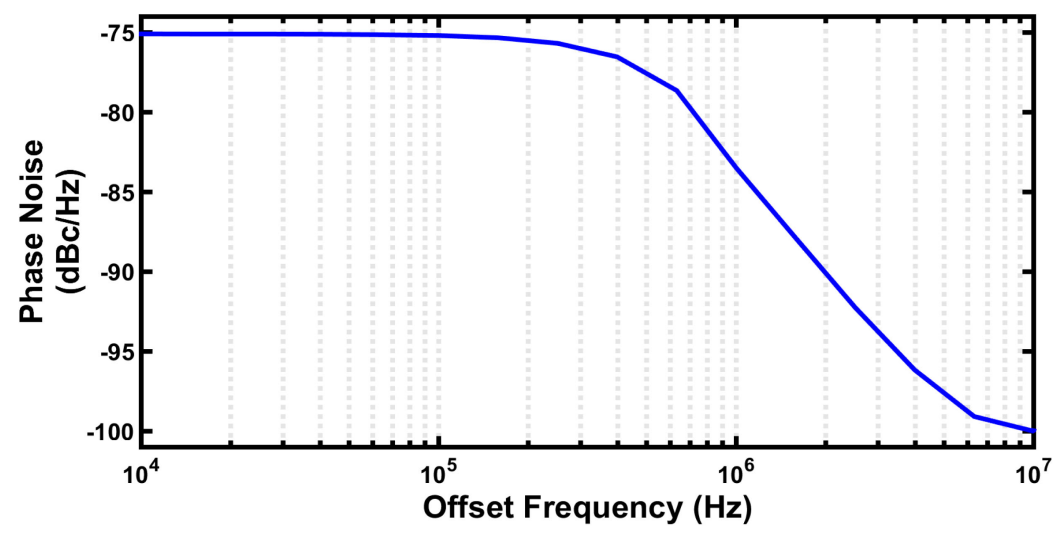

Fig. 8. Phase noise result of rotational DCDL

Table I. Performance summary and comparison with edge-combining DLL

\begin{tabular}{|c|c|c|c|c|c|c|c|}
\hline & This work & \multicolumn{3}{|c|}{ [9] } & [11] & [12] & [13] \\
\hline Process & $180 \mathrm{~nm}$ & \multicolumn{3}{|c|}{$90 \mathrm{~nm}$} & $180 \mathrm{~nm}$ & $350 \mathrm{~nm}$ & $180 \mathrm{~nm}$ \\
\hline Area & $0.608 \mathrm{~mm}^{2}$ & \multicolumn{3}{|c|}{$0.04 \mathrm{~mm}^{2}$} & $0.15 \mathrm{~mm}^{2}$ & $0.601 \mathrm{~mm}^{2}$ & $0.043 \mathrm{~mm}^{2}$ \\
\hline Supply & $1.2 \mathrm{~V}$ & \multicolumn{3}{|c|}{$1.2 \mathrm{~V}$} & $1.8 \mathrm{~V}$ & $3.3 \mathrm{~V}$ & $1.8 \mathrm{~V}$ \\
\hline $\begin{array}{c}\text { Multiplying } \\
\text { Factor }\end{array}$ & 16 & 2 & 3 & 6 & 14 & 4 & 313 \\
\hline $\begin{array}{c}\text { Output } \\
\text { Frequency }\end{array}$ & $16 \mathrm{MHz}$ & $1.7 \mathrm{GHz}$ & $2.55 \mathrm{GHz}$ & $5.1 \mathrm{GHz}$ & $1.4 \mathrm{GHz}$ & $40 \mathrm{MHz}$ & $10 \mathrm{MHz}$ \\
\hline $\begin{array}{c}\text { Reference } \\
\text { Spur }\end{array}$ & $-38 \mathrm{dBc}$ & $-39.2 \mathrm{dBc}$ & $-36.8 \mathrm{dBc}$ & $-26.2 \mathrm{dBc}$ & $-40 \mathrm{dBc}$ & N.A. & $-37.1 \mathrm{dBc}$ \\
\hline $\begin{array}{c}\text { Phase Noise } \\
(\mathrm{dBc} / \mathrm{Hz})\end{array}$ & $\begin{array}{c}-83.5 \\
\text { (a) } 1 \mathrm{MHz}\end{array}$ & $\begin{array}{c}-129.7 \\
\text { (a) } 1 \mathrm{MHz}\end{array}$ & $\begin{array}{c}-127.4 \\
@ 1 \mathrm{MHz}\end{array}$ & $\begin{array}{c}-121.4 \\
@ 1 \mathrm{MHz}\end{array}$ & $\begin{array}{c}-120.1 \\
\text { (a) } 1 \mathrm{MHz}\end{array}$ & N.A. & $\begin{array}{c}-100 \\
\text { (a) } 1 \mathrm{MHz}\end{array}$ \\
\hline RMS Jitter & $1.76 \%$ & & N.A. & & $0.21 \%$ & $3.5 \%$ & $2.3 \%$ \\
\hline Power & $169.4 \mathrm{uW}$ & & $20 \mathrm{~mW}$ & & $20 \mathrm{~mW}$ & $700 \mathrm{uW}$ & $42 \mathrm{uW}$ \\
\hline
\end{tabular}

*exclude off-chip loop filter

\section{Conclusion}

This paper presents a novel architecture of DLL-based clock multiplier for spur reduction. The rotational DCDL and PRNG based randomized delay line selection technique are proposed to reduce the spurs from delay cell mismatches. In the postlayout verification, the proposed technique achieves $5 \sim 11 \mathrm{~dB}$ spur level reduction including $6.2 \mathrm{~dB}$ improvement from the worst case spur. The proposed clock multiplier is implemented in $0.18 \mu \mathrm{m}$ CMOS process, occupies $0.608 \mathrm{~mm}^{2}$ and consumes $169.4 \mu \mathrm{W}$ for $16 \mathrm{MHz}$ generation.

\section{Acknowledgements}

This work was supported by and the National Research Foundation of Korea grant funded by the Korea government (No. 2015R1C1A1A01051634). The chip fabrication and EDA tool were supported by IDEC, Korea. 